\title{
TRANSPOSITION OF A MEMBER OF A DUPLICATED REGION IN MAIZE
}

\author{
G. GAYAZZI \\ Institute of Genetics, University of Milan, Milan, Italy
}

Received 5.vi.75

\begin{abstract}
SUMMARY
A case of transposition of the proximal member of the $R$ duplication, referred to in the text as " case 2 ", is described. The transposed segment is marked with the $P$ component of $R$. $P$, in its new position, maps about 5 units to the right of the $R$ locus.

The recombination values in the region proximal to $P$ of a " case 2 " strand are significantly lower than in normal strands. Plants homozygous for the transposed duplication have an abnormal morphology consisting of narrow leaves and poorly developed male and female inflorescences. Evidence was obtained that the morphogenetic effect is associated with the distal portion of the transposed segment. A recombinational analysis of the "case $2 "$ strand has disclosed the existence of a gene controlling silk pigmentation closely linked to the $P$ duplication. There is circumstantial evidence that the transposed $P$ tends to be lost but the mechanism responsible for this chromosomal instability is unknown.
\end{abstract}

\section{INTRODUGTION}

ThE $R$ locus in maize, one of the complementary factors required for anthocyanin biosynthesis located on the long arm of chromosome 10, has some features of a gene complex. Gene complex is the term given to a chromosomal region resolvable into a series of genes with separate but related functions. It can be used as a model system to understand the structural and functional organisation of gene clusters in eucaryotic cells. Many alleles are known at the locus that differ widely in their organisation (Sastry, 1970; Kermicle, 1973). $R^{r}$ is the symbol used to describe an allele, known as standard $R$, that controls anthocyanin pigmentation in both plant and seed. Stadler and Nuffer (1953) first suggested that $R^{r}$ is a chromosomal region physically divisible into two functionally distinct elements, designated $P$ and $S$, controlling plant and seed pigmentation respectively. Dooner and Kermicle (1971) proved that $P$ and $S$ are differentiated components of a duplicated region and established the relative position of these two genes within the two members of the duplication. Gavazzi and Calati (1972) while studying intralocus recombination in $R^{s t} r^{r}$ heterozygotes $\left(R^{s t}\right.$ conditions green plant and stippled aleurone, $r^{r}$ red plant and colourless aleurone) isolated non-parental seedlings carrying properties of both parental markers, i.e. stippled aleurone and red plant tissues.

The exceptional seedlings, produced with a frequency of about $2 \cdot 69$ $\times 10^{-4}$, proved to have a non-parental strand carrying $P$ linked to the stippled factor $\left(R^{s t}\right)$. Further studies indicated that the frequency of $P-S$ recombination in plants carrying the nonparental strands is unexpectedly 
high, as much as 200 times higher than the frequency of the event that originally gave rise to the exceptional strands. Four of them have been tested and they all exhibit this unexpected increase. In addition three of them produce, when homozygous, short plants with narrow leaves and poorly developed male and female inflorescences. One of the isolates, referred to as case 2 was analysed more extensively than the others to elucidate the genetic basis of the exceptional increase in recombination. The results obtained are reported here.

\section{MATERIAL}

List of markers used:

Mp: $\quad$ Modulator of $P$; a controlling element causing instability of $P$ (pericarp colour).

$R^{s t}\left(p S^{s t}\right)$ : Stippled aleurone, green plant; paramutagenic.

$R^{s k}\left(p S^{s k}\right)$ : A derivative of stippled with a smoky pattern of aleurone variagation, green plant; paramutagenic.

$R^{s c}\left(p S^{s c}\right)$ : A derivative of stippled with self-coloured aleurone and green plant (see Kermicle, 1970, for an account on the genetic basis of self-coloured derivatives).

$r(P s)$ : Colourless aleurone; red plant.

$r^{g}(p s)$ : Colourless aleurone; green plant.

$g: \quad$ Golden plumule and dark yellow silks.

$M^{s t}$ : $\quad$ A stippled modifier that affects specifically the expression of $R^{s t}$ by enhancing the number of dark spots determined by $R^{s t}$. Active both in cis and trans. Its loss leads to a light stippled phenotype.

K10: A large heterochromatic segment attached to the end of the long arm of chromosome 10. In individuals heterozygous for $K 10$, the abnormal 10 is recovered in 70 per cent of the functioning megaspores (preferential segregation, Rhoades, 1942).

Symbols in brackets refer to the dual structure of the $R$ locus. The lower case letters $p$ and $s$ as used in this context, do not distinguish between presence of a recessive allele or absence of the gene component.

All the markers used have the genetic background of the inbred line W22. The order of the genes and their map distances are given below:

$$
\begin{array}{llllllll}
g & 14 & R & 5,7 & M^{s t} & >36 & K
\end{array}
$$

\section{Methods}

"Case 2 " strand was originally isolated as a $g P S^{s k} M^{s t} / G p s m^{s t}$ putative recombinant from a $G P s m^{s t} K / g p S^{s k} M^{s t}$ female test crossed to a homozygous $G p s m^{s t}$ line (Gavazzi and Calati, 1972). The plant has red anthers and silks. The scheme of isolation and progeny test of non-parental strands recovered from plants heretozygous for " case 2 " strand is outlined in fig. 1. Plants of (A) and (B) genotypes were crossed with a homozygous $G p s m^{s t}$ and $g p s m^{s t}$ tester respectively. Seeds produced from these matings were germinated and the seedlings scored for pigment production. Individuals with non-parental genotype (i.e. red seedling and colourless aleurone or 
green seedling and smoky aleurone from (A) parents; red seedling and selfcoloured aleurone or green seedling and smoky aleurone from (B) parents) were transferred to the field where their anther and silk colour was recorded and progeny tested to ascertain the validity of the screening procedure.

(A)

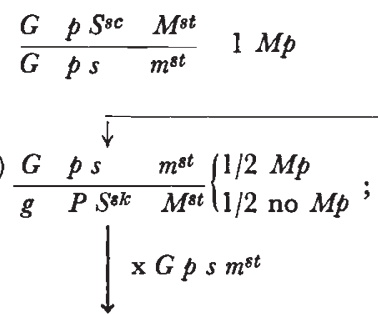

Selected, upon germination, two classes of non-parental seedlings:

(i) $\frac{(\text { ) } P s()}{G \quad p s m^{8 t}}$

(ii)

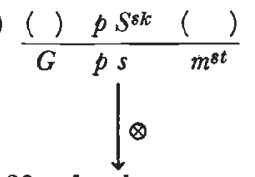

$$
\begin{array}{lll}
g & P S^{s k} & M^{s t} \\
\hline G & p s & m^{s t}
\end{array}
$$

(B) $\frac{G \quad p S^{s c}}{g \quad P S^{s k}} \frac{M^{s t}}{M^{s t}}\left\{\begin{array}{l}1 / 2 M p \\ 1 / 2 \text { no } M p\end{array}\right.$

\begin{tabular}{l}
$\downarrow \mathrm{x} g \rho s$ \\
$S^{s c} M^{s t}$ \\
\hline$s \quad m^{s t}$
\end{tabular}

\begin{tabular}{lll} 
( ) & $P S^{s c}$ & $M^{s t}$ \\
\hline$g$ & $p s$ & $m^{s t}$
\end{tabular}

\begin{tabular}{lll}
() & $p S^{s k}$ & $M^{s t}$ \\
\hline$g$ & $p s$ & $m^{s t}$
\end{tabular}

15-20 colourless or smoky seeds in the selfed progeny of (i) and (ii) respectively, were planted. Mature plants were crossed with an $M^{s t}$ tester line $\left(g p S^{s t} m^{s t}\right)$. In case of progeny of (i) only plants with red anthers were crossed.

FIG. 1.-Crosses designed to detect recombinants in the $R$ region and to establish their flanking marker constitution. Brackets indicate that the gene marker constitution is to be ascertained.

\section{(i) Establishing the outside marker constitution of nonparental strands}

(a) Proximal marker (g)

Determination of the proximal marker $(g)$ constitution of non-parental strands isolated from test-crosses of (A) plants with $G p s m^{s t}$ males required a further generation of selfing while in test-crosses of (B) heterozygotes with $g p s m^{s t}$ males, the constitution was established directly by classifying green vs golden.

(b) Distal marker (Mst)

The $M^{\text {st }}$ constitution can be ascertained only on strands of (A) but not (B) parentage, since only the former are heterozygous for $M^{s t}$.

Since $M^{s t}$ affects specifically the expression of the $R^{s t}$ allele, detection of its presence or absence in non-parental strands required the matings outlined below:

(i) $P s$ strands

$P s / p s$ seedlings isolated in the progeny of test-cross (A) were grown and selfed. Kernels from the self-pollination were grown in the field and plants with red anthers (genotypically $P s / P s$ and $P s / p s$ ) were pollinated with an $R^{s t}$ stock not carrying $M^{s t}$ ( $M^{s t}$ tester). Production of ears segregating for dark and light stippled or uniformly dark stippled indicated presence of $M^{s t}$ in the $P s$ strand, while production of ears uniformly light stippled indicated its absence. 
(ii) $p S^{s k}$ strands $p S^{s k} / p s$ seedlings in the progeny of test-cross (A) were grown and selfed; heterozygous $p S^{s k} / p s$ individuals from the selfed ears were grown in the field and crossed with an $M^{s t}$ tester. The presence or absence of about 6 per cent dark stippled seeds from such crosses indicates the respective presence or absence of $M^{s t}$ in the $p S^{s k}$ nonparental strand.

\section{Results}

(i) Tield of nonparental strands in plants of $(A)$ and $(B)$ genotype

An estimate of the frequency of $P-S^{s k}$ recombination in "case 2 " strand was obtained by scoring the seedlings produced by test-crosses of (A) and (B) parents. Non-parental seedlings (see table 1) appeared with a frequency

\section{TABLE 1}

Frequency of exceptional seedlings with a presumed recombinant strand in the progeny of crosses of $\mathrm{p} \mathrm{s} / \mathrm{P} \mathrm{S}^{\mathrm{sk}}(A)$ and $\mathrm{p} \mathrm{S}^{\mathrm{sc}} / \mathrm{P} \mathrm{Ssk}(B)$ females to p s males

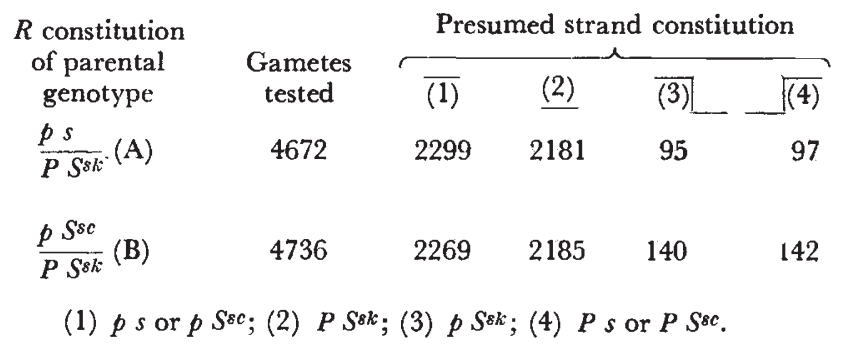

of 4.1 per cent $(192 / 4672)$ and 5.9 per cent $(282 / 4736)$ in the progeny of (A) and (B) parents respectively. The two classes of non-parental seedlings were recovered with approximately the same frequency as one would expect if they resulted from reciprocal exchange. A sample of the exceptional plants was progeny tested to ascertain the validity of the screering procedure.

TABLE 2

Progeny test of the exceptional seedlings isolated as presumed recombinants in the progeny of crosses of females with $(A)$ and $(B)$ genotypes to $\mathrm{p} s$ males

$\begin{array}{cc}\begin{array}{c}R \text { constitution } \\ \text { of parental } \\ \text { genotype }\end{array} & \begin{array}{c}\text { Presumed } \\ \text { recombinants } \\ \text { strands }\end{array} \\ \begin{array}{l}\text { (A) } p s / P S^{s k} \\ p S^{8 k} \\ P s\end{array} \\ \text { (B) } p S^{s c / P S^{8 k}} & \left\{\begin{array}{l}p S^{8 k} \\ P S^{\delta c}\end{array}\right.\end{array}$

\begin{tabular}{|c|c|c|c|}
\hline \multicolumn{4}{|c|}{ Presumed recombinants } \\
\hline Isolated & Tested & Conf. & Non-conf. \\
\hline 95 & 26 & 26 & - \\
\hline 97 & 71 & 71 & - \\
\hline 140 & 53 & 51 & $2\left(P S^{8 k}\right)$ \\
\hline 142 & 69 & 65 & $4\left(P S^{s k}\right)$ \\
\hline
\end{tabular}

The results (table 2) indicate that the procedure is quite effective for the identification of non-parental strands. Only a very few seedlings of (B) parentage appear misclassified. They can be accounted for by one of the following two events :

(a) the lack of pigment development in a $P S^{s k} / p s$ seedling or 
(b) the reversion of smoky to self-coloured occurring during embryo sac development and giving rise to a kernel with coloured endosperm and smoky embryo (see Gavazzi and Colella, 1973 for the origin of self-coloured derivatives during gametophyte development). The former affects the estimate of $p S^{s k}$, the latter of $P S^{s c}$ non-parental strands.

A third factor impeding a correct estimate of the $P S$ recombination in the " case 2 "strand is the reversion of smoky to self-coloured occurring during meiosis of (B) parental females leading to a $P S^{s c}$ strand indistinguishable from a recombinant. The rate of meiotic reversion was estimated in the progeny of (A) females crossed with $p s$ males. It amounts to $0.96 \times 10^{-3}$, a frequency almost negligible when compared to the total frequency of $P S^{s c}$ strands from (B) parents (5.9 per cent).

The frequency of recombination between $P$ and $S^{s k}$, as estimated from the data of table 1 after correction for misclassification, is $4 \cdot 1$ per cent and $5 \cdot 7$ per cent in (A) and (B) genotypes, respectively.

\section{(ii) Allelism test of $\mathrm{P}^{*}$ with standard $\mathrm{P}$}

The unexpectedly high recombination frequency between $P$ and $S^{s k}$ in the "case 2 " strand might have various explanations. Two alternatives appear to be the most likely. One of these ascribes the high recombination rate to an increase in the degree of pairing and exchange in the $R$ region which is related to the presence of $P$ linked to $S^{s k}$ in the " case 2 " strand; the

\begin{tabular}{|c|c|c|c|c|c|c|}
\hline \multirow{3}{*}{$\begin{array}{c}\text { Assumption } \\
P \text { and } P^{*} \text { occupy } \\
\text { different } \\
\text { positions }\end{array}$} & \multicolumn{2}{|c|}{$\begin{array}{l}\text { Parental } \\
\text { genotype (i) }\end{array}$} & \multirow{2}{*}{$\begin{array}{c}\text { Non-parental } \\
\text { strands } \\
\text { \}:p } s s c\end{array}$} & \multicolumn{2}{|c|}{$\begin{array}{l}\text { Parental } \\
\text { genotype (ii) }\end{array}$} & \multirow{3}{*}{$\begin{array}{c}\begin{array}{c}\text { Non-parental } \\
\text { strands }\end{array} \\
\}: p S^{s c} \\
\}: P s P^{*}\end{array}$} \\
\hline & $p S^{s c}$ & $P^{*}$ & & $p S^{s c}$ & $P^{*}$ & \\
\hline & $\overline{p s}$ & & $: p s P^{*}$ & $\overline{P s}$ & & \\
\hline$P$ and $P^{*}$ are & $P^{*}$ & $S_{s c}^{s c}$ & $: P^{*} s$ & $P^{*}$ & $S^{s c}$ & none \\
\hline allelic & $p$ & $s$ & $: p S s c$ & $P$ & $s$ & \\
\hline
\end{tabular}

Frg. 2.-The genetic consequences of reciprocal exchanges between $P^{*}$ and $S^{s c}$ assuming $P^{*}$ either allelic to $P$ or transposed to a new position.

second alternative is based on the assumption that the $P$ component of the "case 2 " strand does not occupy its standard position but is dislocated 4 or 5 units to the right or left of the $R$ locus. To discriminate between the two possibilities, use was made of a $P S^{s c}$ strand isolated as a recombinant from test-crosses of plants of (B) genotype.

Let us refer to the $P$ gene component of the "case 2 " strand as $P^{*}$ to distinguish it from the standard $P$. Genotypes (i) $P^{*} S^{s c} / p s$ and (ii) $P^{*}$ $S^{s c} / P s$ were prepared by pollinating homozygous $P s$ and $p s$ lines with the same pollen parent, $P^{*} S^{s c} / p s$. The predictions, outlined in fig. 2, are that plants of genotype (i) yield two types of non-parental strands under both assumptions, while plants of genotype (ii) yield two types of non-parental strands (only one of the two identifiable) under the first assumption but parental strands only under the second assumption.

The results (table 3 ) fulfil the expectations of the first possibility, thus excluding allelism of the two components. The two cases of non-parental 
$p s$ strands yielded by parents (ii) are accounted for by mutation of $P$ to $p$ in a $P s$ strand. It will be noticed that the yield of $p S^{s c}$ strands $(157 / 1381)$ from parents (i) is significantly higher than that of $p s P^{*}$ strands $(96 / 2188)$. There is evidence (Gavazzi, unpublished) that the excess of the former represents losses of $P^{*}$ not associated with recombination. What remains to be explained is why the frequency of $p S^{s c}$ strands recovered from parents (ii) is almost twice as high as that observed in the case of parents (i) (see last column of table 3 ). It might be that the presence of standard $P$ in the second

TABLE 3

Frequency of parental and exceptional seedlings in the progeny of $\mathrm{P}^{*} \mathrm{Ssc} / \mathrm{p} \mathrm{s}$ and $\mathrm{P} * \mathrm{Ssc} / \mathrm{P} \mathrm{s}$ females crossed to $p$ s males

\begin{tabular}{|c|c|c|c|c|c|c|}
\hline \multirow{3}{*}{$\begin{array}{c}\text { Pistillate } \\
\text { parent } \\
\text { genotype }\end{array}$} & \multicolumn{4}{|c|}{ Presumed strand const. } & \multirow{3}{*}{$\begin{array}{c}\text { Seedlings } \\
\text { scored } \\
3569\end{array}$} & \multirow{3}{*}{$\begin{array}{c}p S^{s c} \\
\text { recomb. } \\
(\%) \\
11.37\end{array}$} \\
\hline & $\begin{array}{c}P^{*} S^{\delta c} \\
\text { (1) }\end{array}$ & $\begin{array}{l}p s \\
(2)\end{array}$ & $\begin{array}{c}P^{*} s \\
(3)\end{array}$ & $\begin{array}{c}p S^{\delta c} \\
(4)\end{array}$ & & \\
\hline & 1224 & 2092 & 96 & 157 & & \\
\hline$P^{*} S^{8 c} P P^{8}$ (ii) & 1153 & $2 \dagger$ & 1620 & 342 & 3117 & $22 \cdot 87$ \\
\hline
\end{tabular}

$\dagger$ Four cases of $p s$ in the progeny of a single ear are considered as a single event.

genotype increases the degree of structural homology thus favouring pairing and recombination. This possibility could be tested with the use of more markers in this chromosomal region.

\section{(iii) $\mathrm{P}^{*}$ and $\mathrm{S}$ sk gene sequence in the "case 2 " strand}

Ascertainment of the outside marker ( $g$ and $M^{s t}$ ) distribution in nonparental strands recovered from test-crosses of (A) and (B) parents should yield information on the gene sequence of $P^{*}$ and $S^{s k}$ in the " case 2 " strand. The mating scheme adopted for this purpose is that outlined in fig. 1. The results of this analysis are presented in the next two sections:

Proximal marker. Non-parental strands of (A) parentage were classified for the $g$ marker as follows:

$$
\frac{P^{*} s}{\frac{G}{60} \frac{g}{4}} \frac{p S^{s k}}{\frac{G}{1} \frac{g}{18}}
$$

If one assumes that $P$ is the proximal and $S$ the distal $R$ component (Stadler and Neuffer, 1953), the data would indicate that the majority of the recombinants (78/83) are associated with a second exchange in the regional proximal to $R$. As to the three-point test-cross data, involving $G p S^{s c} / g P^{*} S^{s k}$ heterozygotes, recombinant strands with an additional exchange in the proximal region comprise 207 out of 232 tested.

The results of this test-cross are shown below:

$$
\frac{P^{*} S^{s k}}{\frac{g}{1616} \frac{G}{191}} \frac{p S^{s c}}{\frac{g}{201} \frac{G}{1708}} \frac{p S^{s k}}{\frac{g}{108} \frac{G}{11}} \frac{P^{*} S^{s c}}{\frac{g}{14} \frac{G}{99}} \begin{aligned}
& 3948 \\
& \text { total }
\end{aligned}
$$

The double exchange interpretation is at variance with the recombinational 
values for the long arm of chromosome 10 reported in the literature. Evidence is in fact available that in this chromosomal region there is positive interference.

Alternatively one could interpret the results by assuming that the order of the $P$ and $S$ components of the " case 2 " strand is inverted. According to this interpretation, the strand constitution together with the map length of each cross-over region, as obtained from the three-point test-cross data, would be represented as follows:

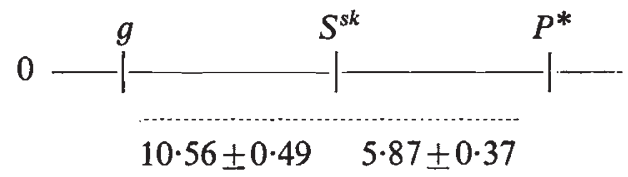

Distal marker. The smoky derivative of stippled carries $M^{\text {st }}$ as distal marker. Its constitution was ascertained in non-parental strands of (A) but not (B) parentage, since only the former are heterozygous for $M^{s t}$. The flanking marker constitution of these strands is as follows:

\begin{tabular}{llllllll}
\multicolumn{4}{c}{$s P^{*}$} & \multicolumn{4}{c}{$p S^{s k}$} \\
\hdashline$G$ & $G$ & $g$ & $g$ & $G$ & $G$ & $g$ & $g$ \\
$M^{s t}$ & $m^{s t}$ & $M^{s t}$ & $m^{\text {st }}$ & $M^{\text {st }}$ & $m^{\text {st }}$ & $M^{\text {st }}$ & $m^{\text {st }}$ \\
14 & 30 & 1 & 1 & 2 & 0 & 14 & 5
\end{tabular}

It can be seen, from these data, that among the strands selected as $S-P^{*}$ cross-overs, 47 had the exchange distally and 20 proximally to $M^{s t}$. Since the $S-P^{*}$ frequency of exchange in this mating (see table 1 ) is $4 \cdot 1$ per cent, $M^{s t}$ must lie between $S^{s k}$ and $P^{*}, 1 \cdot 2$ map units distal to $S^{s k}(20 / 67$ of $4 \cdot 1)$. By combining these data with the previous information the arrangement of the four markers on the "case 2 " strand can be envisaged as follows:

\begin{tabular}{|c|c|c|c|c|}
\hline Linkage map & $g$ & $S^{s k}$ & $M^{s t}$ & $P^{*}$ \\
\hline$\%$ Recombination & $10 \cdot 60$ & & & \\
\hline
\end{tabular}

The recombination values are best interpreted by assuming that the $P$ component of the " case 2 " strand is not lying in its standard position but is dislocated to a new position more than four units distal to the $R$ locus. The data do not allow it to be established whether only the gene $P$ or the entire proximal member of the $R$ duplication has been dislocated to this new position.

The observation that the "case 2 " strand shows a reduction of recombination in the $g_{-} S^{s k}(10.6$ vs 14$)$ and $S^{s k}-M^{s t}(1.22$ vs 5.7) intervals seems to favour the latter possibility.

\section{(iv) Association of the morphogenetic effect with the $\mathrm{P}^{*}$ duplication}

About 25 per cent of the selfed progeny of $p S^{s k} P^{*} / p s$ plants have a reduced growth and abnormal morphology consisting of narrow leaves and poorly developed inflorescences. Similar plants are not found among the colourless seed segregants while they represent the majority of the dark smoky and a minority of the light smoky segregants (see table 4). No abnormal plants are observed in the progeny of sibling plants that have lost $P^{*}$ (see lower part of table 4). Previous tests had shown that about 80 per 
cent of the segregating dark smoky seeds are homozygous for $S^{\text {skt }}$, while more than 95 per cent of the light smoky seeds are heterozygotes. The appearance of abnormal variants is thus associated with homozygosity of the "case 2 " strand or, more specifically, of a region of the chromosome that includes $P^{*}$. To obtain more information on the genetic basis of this morphological

\section{TABLE 4}

Association of the $\mathcal{N} L \dagger$ effect with $\mathrm{P} *$ homozygosity: frequency of $\mathcal{N} L$ plants in the selfed progeny of $\mathrm{p} \mathrm{S} \mathrm{S}^{\text {sk }} \mathrm{P} / \mathrm{p}$ s and $\mathrm{p} \mathrm{S} \mathrm{S}^{\text {sk }} / \mathrm{p}$ s parents

\begin{tabular}{|c|c|c|c|}
\hline $\begin{array}{l}\text { Parental } \\
\text { genotype }\end{array}$ & $\begin{array}{l}\text { Phenotype of } \\
\text { progeny kernels }\end{array}$ & $\begin{array}{l}\text { No. plants } \\
\text { scored }\end{array}$ & $\begin{array}{c}\text { NL plants } \\
(\%)\end{array}$ \\
\hline$p S^{s k} P^{*} / p s$ & $\left\{\begin{array}{l}\text { Dark smoky } \\
\text { Light smoky } \\
\text { Colourless }\end{array}\right.$ & $\begin{array}{l}140 \\
176 \\
117\end{array}$ & $\begin{array}{r}71 \cdot 4 \\
4.5 \\
0 \cdot 0\end{array}$ \\
\hline$p S^{s k} / p S$ & $\left\{\begin{array}{l}\text { Dark smoky } \\
\text { Light smoky } \\
\text { Colorless }\end{array}\right.$ & $\begin{array}{l}44 \\
52 \\
62\end{array}$ & $\begin{array}{l}0 \cdot 0 \\
0 \cdot 0 \\
0 \cdot 0\end{array}$ \\
\hline
\end{tabular}

effect its distribution in the selfed progeny of (A) parents carrying either $p s P^{*}$ or $p S^{s k}$ recombinant strands was established (see fig. 1). The following results were obtained:

\begin{tabular}{|c|c|c|}
\hline $\begin{array}{c}\text { Strand } \\
\text { selected } \\
p S^{s k}\end{array}$ & $\begin{array}{l}\text { Parental } M p \\
\text { constitution } \\
1 \text { or } 0 M p\end{array}$ & $\begin{array}{c}\text { No. strands } \\
\text { analysed } \\
19\end{array}$ \\
\hline$p s P^{*}$ & $1 M p$ & 21 \\
\hline$p s P^{*}$ & $0 M p$ & 27 \\
\hline
\end{tabular}

(The last column shows percentage of progeny segregating, upon selfing, for abnormal variants.)

The results suggest that the morphological effect is accounted for by a chromosomal segment linked to $P^{*}$, presumably identifiable with the proximal member of the $R$ duplication. We refer to the chromosomal determiner of this effect as NL while $\mathrm{NL}^{+}$stands for its absence.

Separation between $P$ and NL was observed only in the $p s P^{*}$ strands derived from (A) parents without $M p$ in their genome. The following $M^{s t}$ and NL distribution among 22 of the 27 strands originally isolated was established:

$$
\begin{aligned}
& \frac{\mathrm{NL}}{M^{s t} m^{s t}} \frac{\mathrm{NL}^{+}}{M^{s t} m^{s t}} \\
& \begin{array}{llll}
2 & 6 & 3 & 11
\end{array}
\end{aligned}
$$

NL could lie on the right or left of $P^{*}$. Assuming NL distal to $P^{*}$ the majority (14/22) of $p s P^{*}$ strands would then be double recombinants, an unlikely possibility. If NL is proximal to $P^{*}$ then it might lie in the $S^{s k}-M^{s t}$ or $M^{s t}-P^{*}$ interval. The expected frequency of $p s P^{*}$ strands carrying NL and $M^{s t}$ or NL only, differs according to the hypothesis, as outlined in fig. 3. The results favour hypothesis 2 . They are best interpreted by assuming that the "case 2 " strand carries a chromosomal segment accounting for the 
abnormal plant morphology when present in double dose. The morphogenetic effect appears to be associated with homozygosity for all or a portion of the chromosomal segment. It is not clear why abnormal plants are not observed in the progeny of $p S^{s k}$ recombinants as well as why, in $p s P^{*}$ strands isolated from parents with $M p, P^{*}$ and NL are always associated. These points will be considered later (see Discussion).

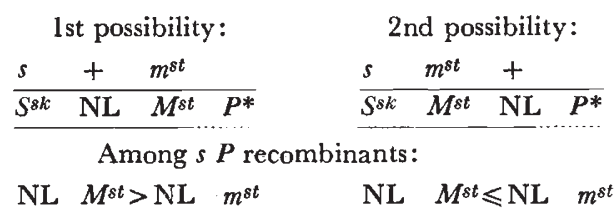

Fig. 3.-Expected NL and $M^{s t}$ constitution of $s P^{*}$ recombinant strands assuming NL either proximal or distal to $M^{s t}$.

\section{(v) Mapping of the Si component}

$S i$ is the symbol used to indicate the $R$ function accounting for pigment production in the silks. Sastry (1970) while studying recombination within the $R^{c h}$ (cherry) complex region first reported that the red silks phene is due to a gene component of cherry mapping between $P$ and $S$. The (A) parental plants used in this work had red silks. The distribution of this character in the non-parental progeny of (A) test-crosses was as follows:

$\begin{array}{lccc}\text { Strand } & \text { Parental } M p & \text { No. strands } & \text { Strands } \\ \text { selected } & \text { constitution } & \text { analysed } & \text { with } S i(\%) \\ p S^{s k} & 1 \text { or } 0 M p & 25 & 0 \cdot 0 \\ p s P^{*} & 1 M p & 23 & 100 \cdot 0 \\ p s P^{*} & 0 M p & 47 & 40.0\end{array}$

The following distribution of $S i$ and $M^{s t}$ markers was observed among the 31 ps $P^{*}$ strands analysed:

$$
\frac{S i}{\frac{M^{s t}}{5} \frac{m^{s t}}{5}} \frac{s i}{\frac{M^{s t}}{2} \frac{m^{s t}}{19}}
$$

The data suggest linkage of $S i$ with $M^{s t}$. Si could map either distally or proximally to $P^{*}$. If distally, 21 out of 31 strands would be double recombinants, an unlikely possibility. If proximally, $S i$ could map in the $S^{s k}-M^{s t}$ or $M^{s t_{-}} P^{*}$ interval. The two possibilities can be distinguished by comparing the frequency of triple cross-over strands expected according to either hypothesis. The strands would be marked Si $m^{s t}$ or $M^{s t} s i$ according to the first and second possibility respectively. The data favour the second possibility, suggesting the sequence $S-M^{s t} t_{-S i}-P$. As in the case of NL, only recombinant strands from (A) parents without $M p$ could be used for this analysis, since no $S i$ segregation was observed in the other sample of $p s P^{*}$ strands derived from (A) parents with $M p$ in their genome. $p S^{s t}$ nonparental strands, on the other hand, were all si. This is interpreted as evidence that the silk colour factor $S i$ requires the presence of an active $P$ for its expression. 
Fig. 4 summarises the observed distribution of the three markers $M^{8 t}$, $S i$ and NL among the $p s P^{*}$ recombinant strands recovered from (A) parents.

Strands analysed

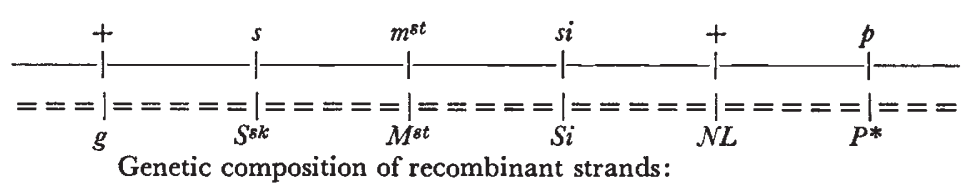

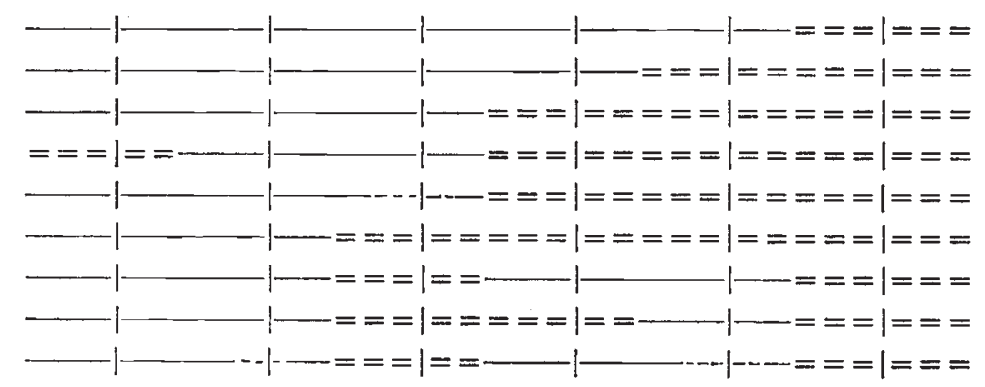

FrG. 4.-Genetic composition of $s P^{*}$ recombinant strands recovered from (A) parents. (When the gene marker composition has not been determined, a dashed line is used.)

The linkage relationship between these markers, estimated on the basis of the values of fig. 4 and assuming the $S^{s t}-P^{*}$ interval equal to 4.11 map units is as follows:

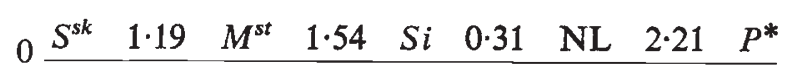

Linkage values should be considered as tentative not only because of the small sample of non-parental strands analysed but also because of the evidence of negative interference in the chromosomal region under study.

\section{Discussion}

The results of " case 2 " strand analyses are best interpreted by assuming that the strand bears the proximal member of the $R$ duplication transposed to a new position. In agreement with this interpretation is the observation that the recombination values of regions adjacent to the transposed duplication are significantly lower in the "case $2 "$ strand than in normal strands. This is in fact to be expected in view of the decreased pairing caused by the duplicated segment that is without counterpart on its homologue. The data however do not allow it to be established whether the proximal member of the duplication alone or adjacent material was involved also in the transposition. The analysis of $p s P^{*}$ and $p S^{s k}$ recombinant strands recovered from $p S^{s k} P^{*} / p s$ heterozygotes discloses additional features:

(i) the $S i$ factor, conditioning red silks, lies between $M^{s t}$ and $P^{*}, 2 \cdot 8$ units proximal to $P^{*}$. The data do not allow it to be established whether $S i$ is located on the $P^{*}$ bearing segment or proximal to it,

(ii) the transposed segment, if present in double dose, upsets normal morphogenesis leading to the appearance of abnormal plants. 
The recombination evidence indicates that the morphogenetic effect is accounted for by a chromosomal segment, referred to as NL, adjacent to $P^{*}$.

The recombination data suggest that NL delimits that portion of the segment whose presence in double dose is required for the morphological effect. The data are in fact best explained (see figure below) by assuming that $p s P^{*}$ strands with an exchange proximal to NL, carry a portion of the segment long enough to allow for the morphological effect. Cross-over $p S^{8 k}$ strands, on the other hand, receive a portion of the segment, proximal to $P^{*}$, too short to cause the morphological effect.

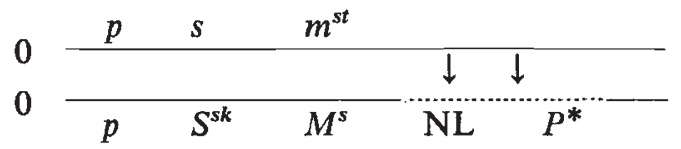

It remains to be explained why, in $p s P^{*}$ strands isolated from (A) parents with $M p, P$ and NL are always associated. This observation suggests the existence of some control of recombination by $\mathrm{Mp}$ resulting in preferential points of breakage within the transposed segment (see Fincham and Sastry, 1974 for a review on the mechanisms of action of controlling elements in maize). The same association between $S i$ and $P$ is found when $p s P^{*}$ strands are analysed, thus making this possibility more plausible.

There is some unpublished evidence that the extent of the chromosomal segment transposed in the four independent cases isolated, is not the same. In all of them the chromosome carrying the transposed segment undergoes frequent losses of its chromosomal material during the mitotic divisions of the endosperm and the sporophytic tissues. Similar results have been reported in haploid strains of Aspergillus nidulans carrying chromosome segments in duplicate (Bainbridge and Roper, 1966). These strains are unstable at mitosis, they undergo frequent intrachromosomal changes involving one or other of the segments present in duplicate. Nga and Roper (1969) termed this instability caused by the imbalance of chromosome segments " mitotic non conformity". The transposed members of tandem duplications are thus a system that it would be interesting to analyse thoroughly in relation to the problem of chromosomal instability.

Acknowledgments. - This research was partially financed by the Italian National Council Research (Rome). I would like to express my appreciation to $\mathrm{Dr} \mathrm{A}$. Ghidoni and D. Mulcahy for their critical reading of the manuscript.

\section{References}

BAINBRIDGE, B. W., AND ROPER, J. A. 1966. Observations on the effects of a chromosome duplication in Aspergillus nidulans. 7. Gen. Microbiol., 42, 417-424.

DOONER, H. K., AND KERMICLE, J. L. 1971. Structure of the $R^{r}$ tandem duplication in maize. Genetics, 67, 427-436.

gincham, J. R. s., AND SASTry, G. R. K. 1974. Controlling elements in maize. Ann. Rev. Genetics, 8, 15-50.

Gavazzi, G., AND CALATI, D. 1972. Study of intralocus events leading to $R$ stippled ( $R^{8 t}$ ) recombinants in Zea mays. Genetica, 43, 489-503.

GAVAZZI, G., AND COLELLA, C. 1973. Different rates of spontaneous reversion of an unstable $R$ allelle $\left(R_{2}^{n c}\right)$ during meiosis and mitotic division of the gametophyte. Mut. Research, 19, 269-271.

KERMICLE, J. L. 1970. Somatic and meiotic instability of $R$-stippled, an aleurone spotting factor in maize. Genetics, 64, 247-258. 
KERMICLE, J. L. 1973. Organization of paramutational components of the $R$ locus in maize. In Basic Mechanisms in Plant Morphogenesis. Brookhaven Symp. Biol., 25, 262-280.

NGA, B. H., AND ROPER, J. A. 1969. A system generating spontaneous intrachromosomal changes at mitosis in Aspergillus nidulans. Genet. Res., 14, 63-70.

RHOAdes, M. M. 1942. Preferential segregation in maize. Genetics, 58, 193-209.

SASTRY, G. R. K. 1970. Paramutation and mutation of $R^{\text {ch }}$ in maize. Theoret. Appl. Genet., 40 , 185-190.

STADLER, L. J., AND NUffer, M. G. 1953. Problems of gene structure. II. Separation of $R^{r}$ elements $(S)$ and $(P)$ by unequal crossing over. Science, 117, 471-472. 